\title{
Chaetomugilins, New Selectively Cytotoxic Metabolites, Produced by a Marine Fish-derived Chaetomium Species
}

\author{
Muroga Yasuhide, Takeshi Yamada, Atsushi Numata, Reiko Tanaka
}

Received: June 20, 2008 / Accepted: September 8, 2008

(C) Japan Antibiotics Research Association

\begin{abstract}
Chaetomugilins $\mathrm{A} \sim \mathrm{F}$ have been isolated from a strain of Chaetomium globosum originally isolated from the marine fish Mugil cephalus, and their absolute stereostructures have been elucidated on the basis of spectroscopic analyses, including 1D and 2D NMR techniques, some chemical transformations and an X-ray analysis. These compounds exhibited significant growth inhibition against cultured P388 cells and HL-60 cells. In addition, chaetomugilins $\mathrm{A}, \mathrm{C}$ and $\mathrm{F}$ showed selective cytotoxic activities against 39 human cancer cell lines.
\end{abstract}

Keywords fungus, marine fish, Chaetomium sp., cytotoxicity, chaetomugilins, COMPARE program

\section{Introduction}

Marine microorganisms are potentially prolific sources of highly bioactive secondary metabolites that might represent useful leads in the development of new pharmaceutical agents. Based on the fact that some of the bioactive materials isolated from marine animals have been produced by bacteria, we have focused our attention on new antitumour materials from microorganisms isolated from marine organisms [1 4]. As part of this study, we have made a search for antitumour compounds from a strain of Chaetomium globosum OUPS-T106B-6 originally obtained from the marine fish Mugil cephalus and found six new cytotoxic metabolites designated as chaetomugilins $\mathrm{A} \sim \mathrm{F}$

T. Yamada (Corresponding author), M. Yasuhide, A. Numata, R. Tanaka: Osaka University of Pharmaceutical Sciences, Nasahara 4-20-1, Takatsuki, Osaka 569-1094, Japan, E-mail: yamada@gly.oups.ac.jp
(1 6) (Fig. 1), which belong to the azaphilones class, from the culture broth of this fungal strain. Azaphilones have various bioactivities such as antimicrobial activity, nitric oxide inhibition (cohaerins) [5], gp120-CD4 binding inhibition (isochromophiliones, ochrephilone, screotiorin and rubrorotiorin) [6], monoamine oxidase inhibition (luteusins, TL-1, TL-2 and chaetoviridins) [7, 8] and platelet-derived growth factor binding inhibition (RP1551s) [9]. The metabolites isolated in this investigation exhibited significant cytotoxic activity against the murine P388 leukemia cell line and the human HL-60 leukemia cell line. The isolation and determination of the absolute stereostructures of chaetomugilins $\mathrm{A} \sim \mathrm{C}(\mathbf{1} \sim \mathbf{3})$ have been briefly described previously [10]. We report herein a detailed study for chaetomugilins $D \sim F \quad(4 \sim 6)$ and the investigation of their cytotoxicities using a disease-oriented panel of 39 human cancer cell lines.

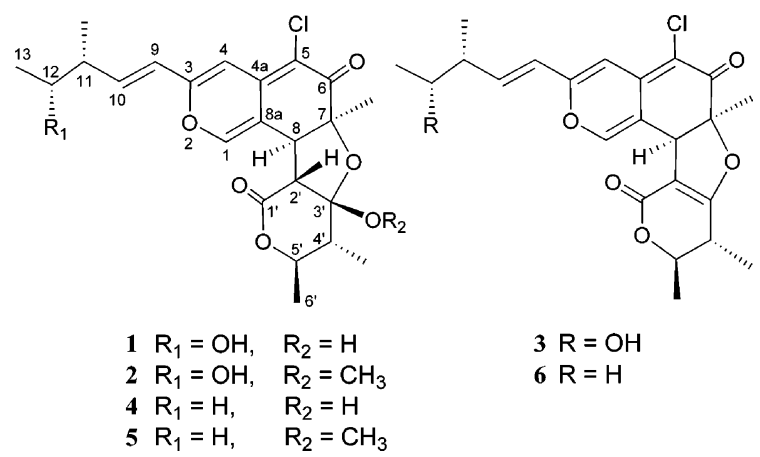

Fig. 1 Structures of chaetomugilins. 
Table 1 Physico-chemical properties of chaetomugilin $D \sim F(\mathbf{4} \sim \mathbf{6})$

\begin{tabular}{|c|c|c|c|}
\hline & 4 & 5 & 6 \\
\hline Appearance & Yellow powder & Yellow powder & Yellow powder \\
\hline Melting point & $95 \sim 97^{\circ} \mathrm{C}$ & $95 \sim 97^{\circ} \mathrm{C}$ & $92 \sim 94^{\circ} \mathrm{C}$ \\
\hline$[\alpha]_{D}^{22}$ & -170.5 (c 0.10, EtOH) & -155.4 (с $0.11, \mathrm{EtOH})$ & -185.1 ( c $0.13, \mathrm{EtOH})$ \\
\hline HRFAB-MS Found: & $435.1557(\mathrm{M}+\mathrm{H})^{+}$ & $449.1735(\mathrm{M}+\mathrm{H})^{+}$ & $417.1461(\mathrm{M}+\mathrm{H})^{+}$ \\
\hline Calcd: & 435.1575 (for $\mathrm{C}_{23} \mathrm{H}_{28} \mathrm{ClO}_{6}$ ) & 449.1731 (for $\mathrm{C}_{24} \mathrm{H}_{30} \mathrm{ClO}_{6}$ ) & 417.1469 (for $\mathrm{C}_{23} \mathrm{H}_{26} \mathrm{ClO}_{5}$ ) \\
\hline Molecular formula & $\mathrm{C}_{23} \mathrm{H}_{27} \mathrm{ClO}_{6}$ & $\mathrm{C}_{24} \mathrm{H}_{29} \mathrm{ClO}_{6}$ & $\mathrm{C}_{23} \mathrm{H}_{25} \mathrm{ClO}_{5}$ \\
\hline$U V \lambda_{\max }(\mathrm{EtOH}) \mathrm{nm}(\log \varepsilon)$ & $283(3.85), 374(3.86), 403$ (3.92) & 290 (3.77), $373(3.85), 400$ (3.90) & $292(3.73), 374(3.74), 410$ (3.82) \\
\hline $\mathrm{IR} v_{\max }(\mathrm{KBr}) \mathrm{cm}^{-1}$ & $3440,1712,1612,1563,1518$ & $1733,1647,1567,1524$ & $1703,1691,1563,1522$ \\
\hline TLC Rf $f^{a}$ & 0.64 & 0.78 & 0.77 \\
\hline $\begin{array}{l}\text { Solubility soluble } \\
\text { insoluble }\end{array}$ & $\begin{array}{l}\text { DMSO, } \mathrm{MeOH}, \mathrm{CHCl}_{3} \\
\mathrm{H}_{2} \mathrm{O}\end{array}$ & $\begin{array}{l}\text { DMSO, } \mathrm{MeOH}, \mathrm{CHCl}_{3} \\
\mathrm{H}_{2} \mathrm{O}\end{array}$ & $\begin{array}{l}\text { DMSO, } \mathrm{MeOH}, \mathrm{CHCl}_{3} \\
\mathrm{H}_{2} \mathrm{O}\end{array}$ \\
\hline
\end{tabular}

${ }^{a}$ Silica gel $\left(10 \% \mathrm{MeOH}\right.$ in $\left.\mathrm{CHCl}_{3}\right)$.

\section{Results and Discussion}

The microorganism from the $M$. cephalus fish was cultured at $27^{\circ} \mathrm{C}$ for 6 weeks in a medium (50 liters) containing $1.0 \%$ soluble starch and $0.1 \%$ casein in $50 \%$ artificial seawater adjusted to $\mathrm{pH} 7.4$ as reported previously [10]. After incubation, the AcOEt extract of the culture filtrate was purified by bioassay-directed fractionation (cytotoxicities against P388 cell line) employing stepwise combination of Sephadex LH-20, silica gel column chromatography and reverse phased HPLC to afford chaetomugilins $A \sim F$ $(\mathbf{1} \sim \mathbf{6})$. The physico-chemical properties of chaetomugilins $\mathrm{D} \sim \mathrm{F}(\mathbf{4} \sim \mathbf{6})$ are summarized in Table 1.

Chaetomugilin D (4) had the molecular formula $\mathrm{C}_{23} \mathrm{H}_{27} \mathrm{ClO}_{6}$ established by the $[\mathrm{M}+\mathrm{H}]^{+}$peak in highresolution fast atom bombardment mass spectrometry (HRFAB-MS) and the ratio of intensity of isotope peaks $\left(\mathrm{MH}^{+} /[\mathrm{MH}+2]^{+}\right)$. Its IR spectrum exhibited bands at 3440, 1712 and $1691 \mathrm{~cm}^{-1}$, characteristic of hydroxyl, ester and conjugated carbonyl groups. A close inspection of the ${ }^{1} \mathrm{H}$ and ${ }^{13} \mathrm{C}$-NMR spectra (Table 2) of $\mathbf{4}$ by DEPT and HMQC experiments revealed the presence of one primary methyl group (C-13), three secondary methyls $\left(11-\mathrm{CH}_{3}, 4^{\prime}-\mathrm{CH}_{3}\right.$ and $\left.\mathrm{C}-6^{\prime}\right)$, one tertiary methyl $\left(7-\mathrm{CH}_{3}\right)$, one methylene (C12), four $s p^{2}$-hybridized methines (C-1, C-4, C-9 and C-10) including an oxygen-bearing carbon (C-1), five $s p^{3}$ methines (C-8, C-11, C-2', C-4' and C-5') including one oxymethine $\left(\mathrm{C}-5^{\prime}\right)$, two quarternary oxygen-bearing $s p^{3}$ carbons (C-7 and $\left.\mathrm{C}-3^{\prime}\right)$ including a hemiacetal carbon (C$\left.3^{\prime}\right)$, four quarternary $s p^{2}$-carbons (C-3, C-4a, C-5 and C-8a) and two carbonyls (C-6 and $\left.\mathrm{C}-1^{\prime}\right)$. The ${ }^{1} \mathrm{H}^{-1} \mathrm{H}$ COSY analysis of $\mathbf{4}$ led to three partial structural units as shown by bold-faced lines in Fig. 2. The geometrical configuration of the double bond moieties (C-9 C-10) was deduced as trans from the coupling constants of the olefinic protons $\left(J_{9,10}=15.5 \mathrm{~Hz}\right)$. The connection of these units and the remaining functional groups was determined on the basis of the key HMBC correlations summarized in Fig. 2. The connection of a chlorine atom to C-5 was reasonable from its chemical shift $\left(\delta_{\mathrm{C}} 110.00\right)$. Thus the planar structure of 4 was elucidated as shown in Fig. 2.

In NOESY experiments of 4, NOE correlations $\left(5^{\prime}-\mathrm{H} / 8\right.$ $\mathrm{H}, 5^{\prime}-\mathrm{H} / 7-\mathrm{CH}_{3}, 2^{\prime}-\mathrm{H} / 4^{\prime}-\mathrm{H}$ and $\left.4^{\prime}-\mathrm{H} / 6^{\prime}-\mathrm{H}\right)$ and the coupling constant $\left(J_{4^{\prime}, 5^{\prime}}=10.2 \mathrm{~Hz}\right)$ implied that $2^{\prime}-\mathrm{H}$ oriented cis to the $3^{\prime}$-hydroxyl group, $4^{\prime}$-H and the $5^{\prime}$-methyl group to be arranged equatorially, and that $8-\mathrm{H}$ is oriented cis to the 7methyl group, and trans to $2^{\prime}-\mathrm{H}$. This result implied that the relative configuration expect at $\mathrm{C}-11$ for 4 is the same as that of $\mathbf{1}$. In addition, the $\mathrm{CD}$ spectrum of $\mathbf{4}$ presented the same curve as that of chaetomugilin A (1), the absolute stereostructure for which had been already deduced (Fig. $3)$. This evidence allowed assignment of the absolute configuration of all the asymmetric centers $\left(7 S, 8 S, 2^{\prime} R\right.$, $3^{\prime} R, 4^{\prime} R$ and $5^{\prime} R$ ) except $C-11$. In order to confirm of the absolute configuration for $\mathrm{C}-11$, which could not be established by NOESY experiments, the degradation of compound $\mathbf{4}$ was carried out. Chromium trioxide oxidation of $\mathbf{4}$ gave $S$-2-methylbutanoic acid, which was identified by comparison of spectral data and specific optical rotations with a commercial sample. Thus the absolute configuration for C-11 was established as $S$.

Chaetomugilin E (5) was assigned the molecular formula $\mathrm{C}_{24} \mathrm{H}_{29} \mathrm{ClO}_{6}$ deduced from HRFAB-MS. The general features of the ${ }^{1} \mathrm{H}$ - and ${ }^{13} \mathrm{C}-\mathrm{NMR}$ spectra (Table 3) closely resembled those of $\mathbf{4}$ except that the proton signal for $\mathrm{H}-\mathbf{4}^{\prime}$ 
Table 2 NMR spectral data of chaetomugilin $\mathrm{D}(\mathbf{4})$ in $\mathrm{CDCl}_{3}$

\begin{tabular}{|c|c|c|c|c|c|c|}
\hline Position & $\delta_{\mathrm{H}}{ }^{a}$ & $\mathrm{~J} / \mathrm{Hz}$ & ${ }^{1} \mathrm{H}-{ }^{1} \mathrm{H}$ COSY & NOE & $\delta_{\mathrm{C}}$ & $\operatorname{HMBC}(C)^{b}$ \\
\hline 1 & $7.28 \mathrm{~s}$ & & & 8 & 145.59 (d) & $3,4 a, 8,8 a$ \\
\hline 3 & & & & & 157.72 (s) & \\
\hline 4 & $6.55 \mathrm{~s}$ & & & 9 & $104.96(d)$ & $3,5,8 a, 9$ \\
\hline $4 a$ & & & & & 140.47 (s) & \\
\hline 5 & & & & & $110(s)$ & \\
\hline 6 & & & & & $189.23(\mathrm{~s})$ & \\
\hline 7 & & & & & 83.93 (s) & \\
\hline 8 & $2.98 \mathrm{~d}$ & $10.0\left(2^{\prime}\right)$ & & $1,7-\mathrm{CH} 3,5^{\prime}$ & $50.49(d)$ & $1,4 a, 6,7,8 a, 7-C H 3,1^{\prime}, 2^{\prime}$ \\
\hline $8 a$ & & & & & 114.26 (s) & \\
\hline 9 & $6.06 \mathrm{~d}$ & $15.5(10)$ & 10 & $4,11,11-\mathrm{CH}_{3}$ & $120.15(d)$ & $3,4,10,11$ \\
\hline 10 & $6.52 \mathrm{dd}$ & $15.5(9), 6.5(11)$ & 9,11 & $11,12,13,11-\mathrm{CH}_{3}$ & $146.90(d)$ & $3,11,12,11-\mathrm{CH}_{3}$ \\
\hline 11 & 2.26 sept & $6.5\left(10,12,11-\mathrm{CH}_{3}\right)$ & $10,12,11-\mathrm{CH}_{3}$ & $9,10,12,13,11-\mathrm{CH}_{3}$ & $38.89(d)$ & $9,10,12,13,11-\mathrm{CH}_{3}$ \\
\hline 12 & $1.43 \mathrm{~m}$ & & 11,13 & $10,11,13,11-\mathrm{CH}_{3}$ & $29.21(t)$ & $10,11,13,11-\mathrm{CH}_{3}$ \\
\hline 13 & $0.91 \mathrm{t}$ & $7.5(12)$ & 12 & $10,11,12$ & $11.68(q)$ & 11,12 \\
\hline 7- $\mathrm{CH}_{3}$ & $1.40 \mathrm{~s}$ & & & $8,4^{\prime}-\mathrm{CH}_{3}, 5^{\prime}$ & $23.27(q)$ & $6,7,8$ \\
\hline $\begin{array}{l}11-\mathrm{CH}_{3} \\
1^{\prime}\end{array}$ & $1.08 d$ & $6.5(11)$ & 11 & $9,10,11,12$ & $\begin{array}{c}19.4(q) \\
170.53(\mathrm{~s})\end{array}$ & $10,11,12$ \\
\hline $2^{\prime}$ & $3.08 d$ & $10.0(8)$ & & & $58.21(d)$ & $8,8 a, 1^{\prime}, 3^{\prime}$ \\
\hline $3^{\prime}$ & & & & & 104.19 (s) & \\
\hline $4^{\prime}$ & $1.90 \mathrm{dq}$ & $10.2\left(5^{\prime}\right), 6.5\left(4^{\prime}-\mathrm{CH}_{3}\right)$ & $5^{\prime}, 4^{\prime}-\mathrm{CH}_{3}$ & $4^{\prime}-\mathrm{CH}_{3}, 6^{\prime}$ & 44.92 (d) & $3^{\prime}, 5^{\prime}, 4^{\prime}-\mathrm{CH}_{3}$ \\
\hline $5^{\prime}$ & $4.30 \mathrm{dq}$ & $10.2\left(4^{\prime}\right), 6.2\left(6^{\prime}\right)$ & $4^{\prime}, 6^{\prime}$ & $8,7-\mathrm{CH}_{3}, 6^{\prime}, 4^{\prime}-\mathrm{CH}_{3}$ & $76.91(d)$ & $3^{\prime}$ \\
\hline $6^{\prime}$ & $1.41 \mathrm{~d}$ & $6.2\left(5^{\prime}\right)$ & $5^{\prime}$ & $4^{\prime}, 5^{\prime}, 4^{\prime}-\mathrm{CH}_{3}$ & $18.69(q)$ & $4^{\prime}, 5^{\prime}$ \\
\hline $4^{\prime}-\mathrm{CH}_{3}$ & $1.13 d$ & $6.5\left(4^{\prime}\right)$ & $4^{\prime}$ & $7-\mathrm{CH}_{3}, 4^{\prime}, 5^{\prime}, 6^{\prime}$ & $8.82(q)$ & $3^{\prime}, 4^{\prime}, 5^{\prime}$ \\
\hline
\end{tabular}

${ }^{a}{ }^{1} \mathrm{H}$ chemical shift values ( $\delta$ ppm from TMS) followed by multiplicity and then the coupling constants $(\mathrm{J} / \mathrm{Hz})$. Figures in parentheses indicate the proton coupling with that position. ${ }^{b}$ Long range ${ }^{1} \mathrm{H}-{ }^{13} \mathrm{C}$ correlations from $\mathrm{H}$ to $\mathrm{C}$ observed in the $\mathrm{HMBC}$ exp.

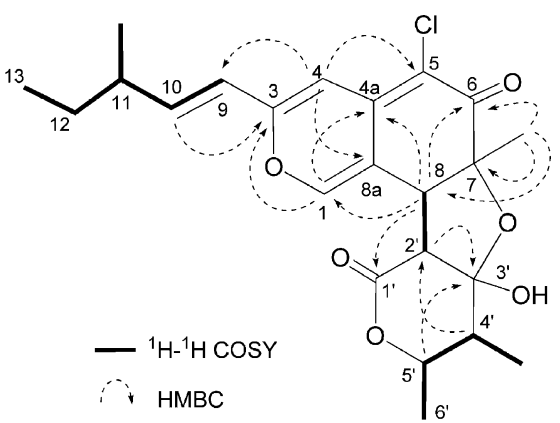

Fig. 2 Selected ${ }^{1} \mathrm{H}-{ }^{1} \mathrm{H}$ COSY and $\mathrm{HMBC}$ correlations in chaetomugilin D (4).

$\left(\delta_{\mathrm{H}} 2.32\right)$ and the carbon signals for $\mathrm{C}-4^{\prime}\left(\delta_{\mathrm{C}} 37.78\right), \mathrm{C}-3^{\prime}$ $\left(\delta_{\mathrm{C}} 106.66\right)$ and $4^{\prime}-\mathrm{CH}_{3}\left(\delta_{\mathrm{C}} 13.52\right)$ in 5 revealed a chemical shift difference relative to those of 4 . In addition, the signal for a methoxyl group $\left(\delta_{\mathrm{H}} 3.25, \delta_{\mathrm{C}} 49.67\right)$ was observed newly. The ${ }^{1} \mathrm{H}-{ }^{1} \mathrm{H}$ COSY and HMBC correlations (from 9$\mathrm{H}$ to $\mathrm{C}-3$, from $4-\mathrm{H}$ to $\mathrm{C}-9$ and $\mathrm{C}-5$, from $1-\mathrm{H}$ to $\mathrm{C}-3, \mathrm{C}-4 \mathrm{a}$, C-8 and C-8a, from 8-H to C-4a, C-6, C-7, 7-CH $\mathrm{CH}_{3}, \mathrm{C}-1^{\prime}$ and

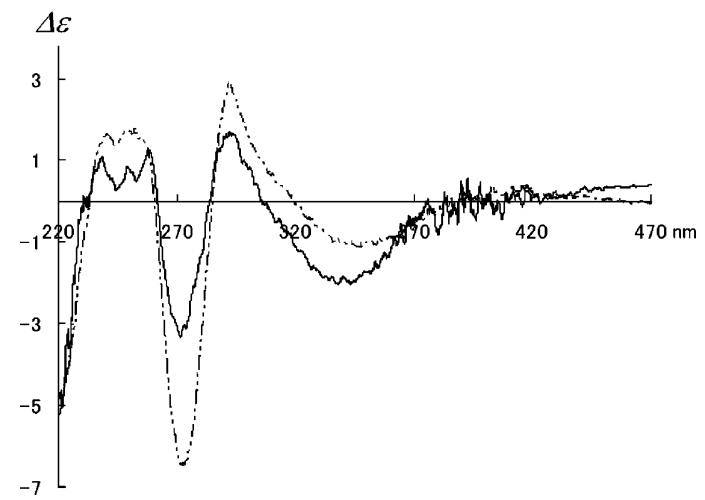

Fig. 3 CD spectra of chaetomugilin $A(\mathbf{1})$ and $D(\mathbf{4})$.

$1,-\cdot-\cdot 4$

$\mathrm{C}-2^{\prime}$, from $5^{\prime}-\mathrm{H}$ to $\mathrm{C}-1^{\prime}$ and $\mathrm{C}-3^{\prime}$, and from the methoxyl group to $\mathrm{C}-3^{\prime}$ ) led to the planar structure for $\mathbf{5}$. In NOESY experiments, NOE correlations $\left(8-\mathrm{H} / 5^{\prime}-\mathrm{H}, 7-\mathrm{CH}_{3} / 4^{\prime}-\mathrm{CH}_{3}\right.$, $8-\mathrm{H} / 4^{\prime}-\mathrm{CH}_{3}$ and $\left.6^{\prime}-\mathrm{H} / 3^{\prime}-\mathrm{OCH}_{3}\right)$ suggested that the relative configulation of $\mathbf{5}$ was the same as that of $\mathbf{4}$. In order to 
Table 3 NMR spectral data of chaetomugilin $\mathrm{E}(\mathbf{5})$ in $\mathrm{CDCl}_{3}$

\begin{tabular}{|c|c|c|c|c|c|c|}
\hline Position & $\delta_{H}{ }^{a}$ & $\mathrm{~J} / \mathrm{Hz}$ & ${ }^{1} \mathrm{H}-{ }^{1} \mathrm{H}$ COSY & NOE & $\delta_{\mathrm{C}}$ & $\mathrm{HMBC}(\mathrm{C})^{\mathrm{b}}$ \\
\hline 1 & $7.50 \mathrm{~s}$ & & & 8 & $145.96(d)$ & $3,4 a, 8,8 a$ \\
\hline 3 & & & & & 157.04 (s) & \\
\hline 4 & $6.49 \mathrm{~s}$ & & & 9 & 104.75 (d) & $3,5,8 a, 9$ \\
\hline $4 a$ & & & & & 139.61 (s) & \\
\hline 5 & & & & & 110.47 (s) & \\
\hline 6 & & & & & $186.84(\mathrm{~s})$ & \\
\hline 7 & & & & & 83.31 (s) & \\
\hline 8 & $3.14 \mathrm{~d}$ & $9.6\left(2^{\prime}\right)$ & $2^{\prime}$ & $1,7-\mathrm{CH}_{3}, 5^{\prime}, 4^{\prime}-\mathrm{CH}_{3}$ & $48.72(d)$ & $1,4 a, 6,7,8 a, 7-\mathrm{CH}_{3}, 1^{\prime}, 2^{\prime}$ \\
\hline $8 a$ & & & & & 114.89 (s) & \\
\hline 9 & $6.04 d$ & $15.5(10)$ & 10 & $4,11,12,11-\mathrm{CH}_{3}$ & $120.33(d)$ & $3,4,10,11$ \\
\hline 10 & $6.48 \mathrm{dd}$ & $15.5(9), 7.1(11)$ & 9,11 & $11,12,13,11-\mathrm{CH}_{3}$ & $145.96(d)$ & $9,12,11-\mathrm{CH}_{3}$ \\
\hline 11 & $2.25 \mathrm{sept}$ & $6.8\left(10,12,11-\mathrm{CH}_{3}\right)$ & $10,12,11-\mathrm{CH}_{3}$ & $9,10,12,13,11-\mathrm{CH}_{3}$ & $38.80(d)$ & $9,10,12,11-\mathrm{CH}_{3}$ \\
\hline 12 & $1.43 \mathrm{~m}$ & & 11,13 & $9,10,11,13,11-\mathrm{CH}_{3}$ & $29.20(t)$ & $10,11,13,11-\mathrm{CH}_{3}$ \\
\hline 13 & $0.90 t$ & $7.5(12)$ & 12 & $10,11,12,11-\mathrm{CH}_{3}$ & $11.67(q)$ & 11,12 \\
\hline $7-\mathrm{CH}_{3}$ & $1.40 \mathrm{~s}$ & & & $8,4^{\prime}-\mathrm{CH}_{3}$ & $23.82(q)$ & $6,7,8$ \\
\hline $\begin{array}{l}11-\mathrm{CH}_{3} \\
1^{\prime}\end{array}$ & $1.08 d$ & $6.8(11)$ & 11 & $9,10,11,12,13$ & $\begin{array}{r}19.42(\mathrm{q}) \\
171.39(\mathrm{~s})\end{array}$ & $10,11,12$ \\
\hline $2^{\prime}$ & $3.02 \mathrm{~d}$ & $9.6(8)$ & 8 & $6^{\prime}, 3^{\prime}-\mathrm{OCH}_{3}$ & $56.92(d)$ & $8,8 a, 1^{\prime}, 3^{\prime}$ \\
\hline $3^{\prime}$ & & & & & $106.66(\mathrm{~s})$ & \\
\hline $4^{\prime}$ & $2.32 \mathrm{qd}$ & $7.1\left(4^{\prime}-\mathrm{CH}_{3}\right), 5.1\left(5^{\prime}\right)$ & $5^{\prime}, 4^{\prime}-\mathrm{CH}_{3}$ & $5^{\prime}, 4^{\prime}-\mathrm{CH}_{3}, 6^{\prime}, 3^{\prime}-\mathrm{OCH}_{3}$ & 37.78 (d) & $3^{\prime}, 4^{\prime}-\mathrm{CH}_{3}$ \\
\hline $5^{\prime}$ & $4.40 \mathrm{qd}$ & $6.8\left(6^{\prime}\right), 5.1\left(4^{\prime}\right)$ & $4^{\prime}, 6^{\prime}$ & $8,4^{\prime}, 6^{\prime}, 4^{\prime}-\mathrm{CH}_{3}$ & $79.28(d)$ & $1^{\prime}, 3^{\prime}, 4^{\prime}$ \\
\hline $6^{\prime}$ & $1.44 \mathrm{~d}$ & $6.8\left(5^{\prime}\right)$ & $5^{\prime}$ & $2^{\prime}, 4^{\prime}, 5^{\prime}, 4^{\prime}-\mathrm{CH}_{3}, 3^{\prime}-\mathrm{OCH}_{3}$ & 19.22 (q) & $4^{\prime}, 5^{\prime}$ \\
\hline $4^{\prime}-\mathrm{CH}_{3}$ & $1.11 \mathrm{~d}$ & $7.1\left(4^{\prime}\right)$ & $4^{\prime}$ & $8,7-\mathrm{CH}_{3}, 4^{\prime}, 5^{\prime}, 6^{\prime}$ & $13.52(q)$ & $3^{\prime}, 4^{\prime}, 5^{\prime}$ \\
\hline $3^{\prime}-\mathrm{OCH}_{3}$ & $3.25 \mathrm{~s}$ & & & $2^{\prime}, 4^{\prime}, 6^{\prime}$ & $49.67(q)$ & $3^{\prime}$ \\
\hline
\end{tabular}

${ }^{\mathrm{a}, \mathrm{b}}$ As in Table 2.

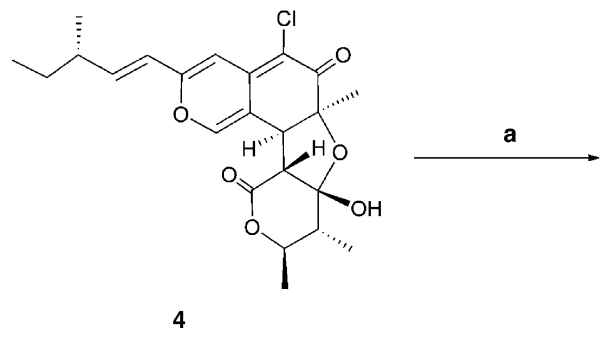

Scheme 1 Reagents and conditions.

a; $\mathrm{MeOH}, p-\mathrm{TsOH}, 20^{\circ} \mathrm{C}, 2$ hours.

determine the absolute stereostructure of $\mathbf{5}$, the methylation of the hydroxyl group at C-3' in $\mathbf{4}$ was carried out to derive of 5 as shown in Scheme 1 as reported previously [10]. The treatment with $p$-TsOH of 4 in $\mathrm{MeOH}$ gave two products 5 and 6. These reaction products were confirmed to be identical with natural substances 5 and $\mathbf{6}$ in IR, UV, NMR spectra and optical rotations. These evidences revealed the absolute stereostructures for chaetomugilins E (5) and F (6) described later. Compound 4 was stable in $\mathrm{MeOH}$ for a week. Therefore this evidence suggests that both $\mathbf{5}$ and $\mathbf{6}$ were not artefacts of 4 .

Chaetomugilin F (6) was assigned the molecular formula $\mathrm{C}_{23} \mathrm{H}_{25} \mathrm{ClO}_{5}$ which contained two hydrogen and one oxygen atoms less than that of 4 . The general features of its UV, IR and NMR spectra (Table 4) closely resembled those of $\mathbf{4}$ except that the proton signals for $8-\mathrm{H}\left(\delta_{\mathrm{H}} 4.22\right), 7-\mathrm{CH}_{3}\left(\delta_{\mathrm{H}}\right.$ $1.72), 2^{\prime}-\mathrm{H}$ (disappearance) and $4^{\prime}-\mathrm{H}\left(\delta_{\mathrm{H}} 2.58\right)$, and the carbon signals for C-7 $\left(\delta_{\mathrm{C}} 91.24\right), \mathrm{C}-8\left(\delta_{\mathrm{C}} 44.73\right), \mathrm{C}-2^{\prime}\left(\delta_{\mathrm{C}}\right.$ 
Table 4 NMR spectral data of chaetomugilin $\mathrm{F}(\mathbf{6})$ in $\mathrm{CDCl}_{3}$

\begin{tabular}{|c|c|c|c|c|c|c|}
\hline Position & $\delta_{\mathrm{H}}{ }^{a}$ & $\mathrm{~J} / \mathrm{Hz}$ & ${ }^{1} \mathrm{H}-{ }^{1} \mathrm{H}$ COSY & NOE & $\delta_{\mathrm{C}}$ & $\mathrm{HMBC}(\mathrm{C})^{\mathrm{b}}$ \\
\hline 1 & $7.90 \mathrm{~s}$ & & & 8 & 147.67 (d) & $3,4 a, 8,8 a$ \\
\hline 3 & & & & & 157.82 (s) & \\
\hline 4 & $6.53 \mathrm{~s}$ & & & 9 & $105(d)$ & $3,5,8 a, 9$ \\
\hline $4 a$ & & & & & $142.32(\mathrm{~s})$ & \\
\hline 5 & & & & & 109.66 (s) & \\
\hline 6 & & & & & 181.95 (s) & \\
\hline 7 & & & & & $91.24(\mathrm{~s})$ & \\
\hline 8 & $4.22 \mathrm{~s}$ & & & $1,7-\mathrm{CH}_{3}, 4^{\prime}-\mathrm{CH}_{3}$ & $44.73(d)$ & $1,4 a, 6,7,8 a, 7-\mathrm{CH}_{3}, 2^{\prime}, 3^{\prime}$ \\
\hline $8 a$ & & & & & $113.91(\mathrm{~s})$ & \\
\hline 9 & $6.04 \mathrm{~d}$ & $15.5(10)$ & 10 & $4,11,11-\mathrm{CH}_{3}$ & $120.18(d)$ & $3,4,10,11$ \\
\hline 10 & $6.52 \mathrm{dd}$ & $15.5(9), 6.5$ (11) & 9,11 & $11,12,13,11-\mathrm{CH}_{3}$ & $146.69(d)$ & $3,9,11,12,11-\mathrm{CH}_{3}$ \\
\hline 11 & 2.25 sept & $6.5\left(10,12,11-\mathrm{CH}_{3}\right)$ & $10,12,11-\mathrm{CH}_{3}$ & $9,10,12,13,11-\mathrm{CH}_{3}$ & $38.83(d)$ & $9,10,12,13,11-\mathrm{CH}_{3}$ \\
\hline 12 & $1.43 \mathrm{~m}$ & & 11,13 & $10,11,13,11-\mathrm{CH}_{3}$ & $29.14(t)$ & $10,11,13,11-\mathrm{CH}_{3}$ \\
\hline 13 & $0.90 \mathrm{t}$ & $7.6(12)$ & 12 & $10,11,12$ & 11.67 (q) & 11,12 \\
\hline 7- $\mathrm{CH}_{3}$ & $1.72 \mathrm{~s}$ & & & $8,4^{\prime}-\mathrm{CH}_{3}$ & $24.92(q)$ & $6,7,8$ \\
\hline $11-\mathrm{CH}_{3}$ & $1.08 d$ & $6.5(11)$ & 11 & $9,10,11,12$ & $19.49(q)$ & $10,11,12$ \\
\hline $1^{\prime}$ & & & & & 164.07 (s) & \\
\hline $2^{\prime}$ & & & & & 101.13 (s) & \\
\hline $3^{\prime}$ & & & & & 174.88 (s) & \\
\hline $4^{\prime}$ & 2.58 quint & $7.0\left(5^{\prime}, 4^{\prime}-\mathrm{CH}_{3}\right)$ & $5^{\prime}, 4^{\prime}-\mathrm{CH}_{3}$ & $5^{\prime}, 4^{\prime}-\mathrm{CH}_{3}, 6^{\prime}$ & $35.04(d)$ & $2^{\prime}, 3^{\prime}, 5^{\prime}, 6^{\prime}, 4^{\prime}-\mathrm{CH}_{3}$ \\
\hline $5^{\prime}$ & 4.29 quint & $7.0\left(4^{\prime}, 6^{\prime}\right)$ & $4^{\prime}, 6^{\prime}$ & $4^{\prime}, 6^{\prime}, 4^{\prime}-\mathrm{CH}_{3}$ & 79.4 (d) & $1^{\prime}, 3^{\prime}, 4^{\prime}, 6^{\prime}, 4^{\prime}-\mathrm{CH}_{3}$ \\
\hline $6^{\prime}$ & $1.40 \mathrm{~d}$ & $7.0\left(5^{\prime}\right)$ & $5^{\prime}$ & $4^{\prime}, 5^{\prime}, 4^{\prime}-\mathrm{CH}_{3}$ & $19.36(q)$ & $4^{\prime}, 5^{\prime}$ \\
\hline $4^{\prime}-\mathrm{CH}_{3}$ & $1.24 d$ & $7.0\left(4^{\prime}\right)$ & $4^{\prime}$ & $8,4^{\prime}, 5^{\prime}, 6^{\prime}, 7^{\prime}-\mathrm{CH}_{3}$ & $14.07(q)$ & $3^{\prime}, 4^{\prime}, 5^{\prime}$ \\
\hline
\end{tabular}

${ }^{\mathrm{a}, \mathrm{b}}$ As in Table 2.

$101.13), \mathrm{C}-3^{\prime}\left(\delta_{\mathrm{C}} 174.88\right), \mathrm{C}-4^{\prime}\left(\delta_{\mathrm{C}} 35.04\right), \mathrm{C}-1^{\prime}\left(\delta_{\mathrm{C}}\right.$ $164.07)$ and $4^{\prime}-\mathrm{CH}_{3}\left(\delta_{\mathrm{C}} 14.07\right)$ in 6 revealed a chemical shift difference relative to those of 4 . The ${ }^{1} \mathrm{H}-{ }^{1} \mathrm{H}$ COSY correlations and the key $\mathrm{HMBC}$ correlations (from 8- $\mathrm{H}$ to $\mathrm{C}-3^{\prime}$, from $4^{\prime}-\mathrm{H}$ to $\mathrm{C}-2^{\prime}$ and $\mathrm{C}-3^{\prime}$, and from $5^{\prime}-\mathrm{H}$ to $\mathrm{C}-1^{\prime}$ and $\mathrm{C}-3^{\prime}$ ) showed that a double bond was present at $\mathrm{C}$ $2^{\prime} \sim \mathrm{C}-3^{\prime}$. The above evidence, together with the molecular formula of 6 , suggested the presence of the ether linkage between $\mathrm{C}-7$ and C-3'. Thus, the planar structure of $\mathbf{6}$ was elucidated. Observed NOEs $\left(4^{\prime}-\mathrm{CH}_{3} / 8-\mathrm{H}\right.$ and $4^{\prime}-\mathrm{CH}_{3} / 7-$ $\mathrm{CH}_{3}$ ) implied that $4^{\prime}-\mathrm{CH}_{3}$ is oriented cis to $8-\mathrm{H}$ and $7-\mathrm{CH}_{3}$ in axial arrangements. In addition, NOE correlation between $4^{\prime}-\mathrm{CH}_{3}$ and $6^{\prime}-\mathrm{H}$ was observed very weakly. Based on the evidence summarized above, the relative configurations of C-7, C-8, C-4' and C-5' in 6 are the same as those of $\mathbf{4}$ and $\mathbf{5}$. Consequently, the absolute stereostructure for $\mathbf{6}$ was established by the derivation from 4.

Chaetomugilins are similar to some of azaphilones reported previously, however it is interesting that compound 3 and $\mathbf{6}$ have the double bond between $\mathrm{C}-2^{\prime}$ and $\mathrm{C}-3^{\prime}$ in tetracyclic ring system.
Table 5 Cytotoxity of the metabolites against P388 and HL-60 cells

\begin{tabular}{|c|c|c|}
\hline Compounds & $\begin{array}{l}\text { Cell line P388 } \\
\qquad \mathrm{IC}_{50}(\mu \mathrm{M})^{\mathrm{a}}\end{array}$ & $\begin{array}{c}\text { Cell line HL-60 } \\
\qquad \mathrm{IC}_{50}(\mu \mathrm{M})^{\mathrm{a}}\end{array}$ \\
\hline Chaetomugilin A (1) & 8.7 & 7.3 \\
\hline$B(\mathbf{2})$ & 18.7 & 16.5 \\
\hline$C(\mathbf{3})$ & 3.6 & 2.7 \\
\hline $\mathrm{D}(\mathbf{4})$ & 7.5 & 6.8 \\
\hline$E(\mathbf{5})$ & 15.7 & 13.2 \\
\hline$F(6)$ & 3.3 & 1.3 \\
\hline $5-F U^{b}$ & 1.7 & 2.7 \\
\hline
\end{tabular}

a DMSO was used for vehicle.

${ }^{\mathrm{b}}$ Positive control.

The growth inhibition assay using the various cancer cell lines is reported rarely for the other azaphilones. As a primary screen for antitumour activities, the cancer cell growth inhibitory properties of chaetomugilins $\mathrm{A} \sim \mathrm{F}(\mathbf{1} \sim \mathbf{6})$ were examined using the murine P388 leukemia cell line 
Table 6 Cytotoxicity of chaetomugilins against a panel of 39 human cancer cell lines

\begin{tabular}{|c|c|c|c|c|c|c|c|}
\hline \multirow{2}{*}{$\begin{array}{l}\text { Origin of } \\
\text { cancer }\end{array}$} & \multirow{2}{*}{ Cell line } & \multicolumn{6}{|c|}{$\log \mathrm{GI}_{50}(\mathrm{M})^{\mathrm{a}}$} \\
\hline & & 1 & 2 & 3 & 4 & 5 & 6 \\
\hline \multirow[t]{5}{*}{ Breast } & $\mathrm{HBC}-4$ & $-4.00^{\mathrm{e}}$ & -4.22 & -4.82 & -4.00 & -4.59 & -6.43 \\
\hline & BSY-1 & -4.00 & -4.64 & -5.54 & -4.55 & -4.75 & -5.63 \\
\hline & HBC-5 & -4.00 & -4.56 & -4.56 & -4.36 & -4.67 & -5.19 \\
\hline & MCF-7 & -4.43 & -4.16 & -5.00 & -4.08 & -4.44 & -6.14 \\
\hline & MDA-MB-231 & -4.00 & -4.51 & -4.48 & -4.00 & -4.60 & -5.30 \\
\hline Central & $U-251$ & -4.00 & -4.00 & -4.78 & -4.00 & -4.55 & -5.50 \\
\hline nervous & SF-268 & -4.00 & -4.05 & -4.63 & -4.00 & -4.59 & -5.37 \\
\hline \multirow[t]{4}{*}{ system } & SF-295 & -4.00 & -4.00 & -4.89 & -4.00 & -4.51 & -5.85 \\
\hline & SF-539 & -4.00 & -4.25 & -5.37 & -4.00 & -4.72 & -6.66 \\
\hline & SNB-75 & -4.36 & -4.21 & -5.43 & -4.34 & -4.58 & -5.59 \\
\hline & SNB-78 & -4.00 & -4.15 & -4.70 & -4.14 & -4.68 & -5.26 \\
\hline \multirow[t]{5}{*}{ Colon } & HCC2998 & -4.00 & -4.00 & -4.78 & -4.00 & -4.63 & -4.83 \\
\hline & KM-12 & -4.00 & -4.08 & -4.68 & -4.00 & -4.64 & -6.21 \\
\hline & HT-29 & -4.00 & -4.01 & -4.51 & -4.00 & -4.64 & -5.02 \\
\hline & HCT-15 & -4.00 & -4.14 & -4.65 & -4.00 & -4.60 & -5.29 \\
\hline & HCT-116 & -4.00 & -4.31 & -5.33 & -4.00 & -4.72 & -5.61 \\
\hline \multirow[t]{7}{*}{ Lung } & $\mathrm{NCl}-\mathrm{H} 23$ & -4.00 & -4.00 & -4.51 & -4.00 & -4.59 & -4.83 \\
\hline & $\mathrm{NCl}-\mathrm{H} 226$ & -4.18 & -4.00 & -4.51 & -4.00 & -4.64 & -5.26 \\
\hline & $\mathrm{NCl}-\mathrm{H} 522$ & -4.36 & -4.81 & -5.38 & -4.40 & -4.78 & -5.67 \\
\hline & $\mathrm{NCl}-\mathrm{H} 460$ & -4.00 & -4.00 & -4.68 & -4.00 & -4.44 & -5.23 \\
\hline & A549 & -4.00 & -4.00 & -4.71 & -4.00 & -4.38 & -5.14 \\
\hline & DMS273 & -4.00 & -4.00 & -5.30 & -4.00 & -4.66 & -6.35 \\
\hline & DMS114 & -4.06 & -4.11 & -4.79 & -4.00 & -4.84 & -5.48 \\
\hline Melanoma & LOX-IMVI & -4.15 & -4.24 & -4.81 & -4.00 & -4.71 & -5.34 \\
\hline \multirow[t]{5}{*}{ Ovary } & OVCAR-3 & -4.00 & -4.61 & -4.96 & -4.00 & -4.67 & -5.51 \\
\hline & OVCAR-4 & -4.00 & -4.18 & -4.36 & -4.00 & -4.72 & -4.69 \\
\hline & OVCAR-5 & -4.00 & -4.13 & -4.40 & -4.00 & -4.61 & -5.39 \\
\hline & OVCAR-8 & -4.00 & -4.00 & -4.71 & -4.00 & -4.54 & -5.11 \\
\hline & SK-OV-3 & -4.00 & -4.00 & -5.20 & -4.00 & -4.38 & -5.47 \\
\hline \multirow[t]{2}{*}{ Kidney } & RXF-631L & -4.00 & -4.00 & -4.47 & -4.00 & -4.52 & -5.14 \\
\hline & $\mathrm{ACHN}$ & -4.00 & -4.19 & -4.73 & -4.00 & -4.67 & -5.33 \\
\hline \multirow[t]{6}{*}{ Stomach } & St-4 & -4.00 & -4.00 & -5.03 & -4.00 & -4.54 & -4.98 \\
\hline & MKN1 & -4.00 & -4.52 & -5.23 & -4.00 & -4.74 & -5.55 \\
\hline & MKN7 & -4.00 & -4.35 & -4.78 & -4.00 & -4.67 & -5.40 \\
\hline & MKN28 & -4.73 & -4.22 & -4.52 & -4.00 & -4.47 & -5.32 \\
\hline & MKN45 & -4.51 & -4.00 & -5.00 & -4.00 & -4.49 & -5.28 \\
\hline & MKN74 & -5.24 & -4.34 & -4.61 & -4.00 & -4.60 & -5.49 \\
\hline \multirow[t]{2}{*}{ Prostate } & DU-145 & -4.00 & -4.00 & -4.63 & -4.00 & -4.52 & -5.42 \\
\hline & PC-3 & -4.00 & -4.41 & -4.99 & -4.13 & -4.68 & -5.27 \\
\hline MG-MIDb & & -4.10 & -4.19 & -4.83 & -4.05 & -4.61 & -5.45 \\
\hline Delta ${ }^{c}$ & & 1.13 & 0.62 & 0.71 & 0.50 & 0.23 & 1.21 \\
\hline Range $^{d}$ & & 1.24 & 0.81 & 1.19 & 0.55 & 0.46 & 1.97 \\
\hline
\end{tabular}

${ }^{a}$ Log concentration of compounds for inhibition of cell growth at $50 \%$ compared to control. ${ }^{b}$ Mean value of log $\mathrm{Gl}_{50}$ over all cell lines tested. ${ }^{\mathrm{c}}$ The difference in $\log \mathrm{GI}_{50}$ value of the most sensitive cell and MG-MID value. ${ }^{d}$ The difference in log $\mathrm{GI}_{50}$ value of the most sensitive cell and the least sensitive cell. ${ }^{e}$ It is set expediently as the value of the least sensitive cell to calculate the MG-MID. 
and the human HL-60 leukemia cell line and a diseaseoriented panel of 39 human cancer cell lines (HCC panel) $[11,12]$. Compounds $\mathbf{3}$ and $\mathbf{6}$ exhibited significant cytotoxic activity against P388 and HL-60 cell lines equal to 5-fluoro uracil (Table 5). These results implied that the presence of the hydroxyl group at C-12 and the methoxyl group at $\mathrm{C}-3^{\prime}$ reduce the activity a little. In addition, these compounds were evaluated for growth inhibition against 39 human cancer cell lines. Among them, compound 6 exhibited remarkable growth inhibition (the MG-MID -5.45 , effective value: $\leq-5.0$ ) (Table 6). Furthermore, the delta and range values of $\mathbf{6}$ were 1.21 and 1.97, respectively (effective value: delta $\geq 0.5$ as well as range $\geq 1.0$ ), disclosing that this compound showed selective cytotoxic activity. Whereas MG-MID of other compounds were moderate, however, the delta and range values of $\mathbf{1}$ (1.13 and 1.24 , respectively) and 3 ( 0.71 and 1.19 , respectively) showed more potency. Evaluation of the pattern of differential cytotoxicity using the COMPARE program [11, 12] suggested the possibility that the mode of action for $\mathbf{1}$, 3 and $\mathbf{6}$ might be different to that shown by any other anticancer drug developed to date.

\section{Experimental}

\section{General}

Mps were determined on a Yanagimoto micro-melting point apparatus and are uncorrected. UV spectra were recorded on a Hitachi U-2000 spectrophotometer and IR spectra on a JASCO FT/IR-680 plus. NMR spectra were recorded at $27^{\circ} \mathrm{C}$ on Varian UNITY INOVA-500 and MERCURY spectrometers with TMS as an internal reference. FAB-MS were obtained using a JOEL JMS-700 (Ver. 2) mass spectrometer. Optical rotations were recorded on a JASCO J-820 polarimeter. Liquid chromatography over silica gel (mesh 230 400) was performed at medium pressure. HPLC was run on a Waters ALC-200 instrument equipped with a differential refractometer ( $\mathrm{R} 401)$ and Shim-pack PREP-ODS $(25 \mathrm{~cm} \times 20 \mathrm{~mm}$ i.d. $)$. Analytical TLC was performed on precoated Merck aluminium sheets (DC-Alufolien Kieselgel 60 F254, 0.2 mm) with the solvent system $\mathrm{CH}_{2} \mathrm{Cl}_{2}-\mathrm{MeOH}(9: 1)$, and compounds were viewed under UV lamp and sprayed with $10 \% \mathrm{H}_{2} \mathrm{SO}_{4}$ followed by heating.

\section{Culturing and Isolation of Metabolites}

A strain of Chaetomium globosum was initially isolated from the marine fish Mugil cephalus, collected in the Katsuura bay of Japan in October, 2000. The marine fish was wiped with EtOH and its gastrointestinal tract applied to the surface of nutrient agar layered in a Petri dish. Serial transfers of one of the resulting colonies provided a pure strain of C. globosum. The fungal strain was cultured at $27^{\circ} \mathrm{C}$ for six weeks in a liquid medium (50 liters) containing soluble starch $1.0 \%$ and casein $0.1 \%$ in $50 \%$ artificial seawater adjusted to $\mathrm{pH} 7.5$. The culture was filtered under suction and the mycelia collected were extracted thrice with $\mathrm{MeOH}$. The combined extracts were evaporated in vacuo to give a mixture of crude metabolites $(23.8 \mathrm{~g})$, the $\mathrm{CHCl}_{3}-\mathrm{MeOH}(1: 1)$-soluble fraction of which exhibited cytotoxicity. The culture filtrate was extracted thrice with EtOAc. The combined extracts were evaporated in vacuo to afford a mixture of crude metabolites $(10.21 \mathrm{~g})$, which exhibited cytotoxicity $\left(\mathrm{ED}_{50}\right.$ $35.8 \mu \mathrm{g} / \mathrm{ml}$ ). The EtOAc extract was passed through Sephadex $\mathrm{LH}-20$, using $\mathrm{CHCl}_{3}-\mathrm{MeOH}(1: 1)$ as the eluent. The second fraction $(3.4 \mathrm{~g})$, in which the activity was concentrated, was chromatographed on a silica gel column with a $\mathrm{CHCl}_{3}-\mathrm{MeOH}$ gradient as the eluent. The $\mathrm{CHCl}_{3}$ eluate $(821.5 \mathrm{mg}$ ) was purified by HPLC using $\mathrm{MeCN}$ $\mathrm{H}_{2} \mathrm{O}(80: 20)$ as the eluent to afford $4(52.2 \mathrm{mg}), \mathbf{5}(5.3 \mathrm{mg})$ and $6(4.9 \mathrm{mg})$. The $\mathrm{MeOH}-\mathrm{CHCl}_{3}(1: 99)$ eluate (716.52 mg) was purified by HPLC using $\mathrm{MeOH}-\mathrm{H}_{2} \mathrm{O}$ $(70: 30)$ as the eluent to afford two fractions Fr. $1(85.9 \mathrm{mg})$ and Fr. $2(32.27 \mathrm{mg})$, both of which exhibited cytotoxicity. Fr. 1 was purified by HPLC using $\mathrm{MeOH}-\mathrm{H}_{2} \mathrm{O}(60: 40)$ as the eluent to afford $1(72.2 \mathrm{mg})$. Fr. 2 was purified by HPLC using $\mathrm{MeCN}-\mathrm{H}_{2} \mathrm{O}(55: 45)$ as the eluent to afford 2 (7.7 mg) and $\mathbf{3}$ (10.2 mg).

\section{Derivatization of 5 and $\mathbf{6}$ from 4}

$p$-TsOH $(8.2 \mathrm{mg})$ was added to a $\mathrm{MeOH}$ solution $(5.0 \mathrm{ml})$ of chaetomugilin D (1) (32.7 mg), and the reaction mixture was left at room temperature for 3 hours. The solvent was evaporated off under reduced pressure, and the residue was purified by HPLC using $\mathrm{MeCN}-\mathrm{H}_{2} \mathrm{O}(80: 20)$ as the eluent to afford $\mathbf{5}(9.2 \mathrm{mg})$ and $\mathbf{6}(7.1 \mathrm{mg})$.

\section{Chromium Trioxide Oxidation of 4}

$\mathrm{CrO}_{3}(200 \mathrm{mg})$ was added to compound $4(97.6 \mathrm{mg})$ dissolve in $\mathrm{H}_{2} \mathrm{O}(0.2 \mathrm{ml}), \mathrm{H}_{2} \mathrm{SO}_{4}(0.2 \mathrm{ml})$ and $\mathrm{AcOH}(5.0$ $\mathrm{ml}$ ), and the reaction mixture was left at room temperature for 22 hours. The solvent was extracted with EtOAc and the extract was evaporated off under reduced pressure. The residue was purified by HPLC using $\mathrm{MeCN}-\mathrm{H}_{2} \mathrm{O}(55: 45)$ as the eluent to afford 2-methylbutanoic acid $(2.7 \mathrm{mg})$ as a colorless oil, $[\alpha]_{\mathrm{D}} 23(c 0.23$, EtOH). This acid was identified with a commercial sample of $(S)$-2-methylbutanoic acid in ${ }^{1} \mathrm{H}-\mathrm{NMR}$ spectrum and optical rotation. 


\section{Cytotoxic Assay agaist P388 and HL-60 Cell Lines}

Cytotoxic activities of chaetomugilins $\mathrm{A} \sim \mathrm{F}(\mathbf{1} \sim \mathbf{6})$ were examined by 3-(4,5-dimethyl-2-thiazolyl)-2,5-diphenyl-2Htetrazolium bromide (MTT) method. P388 and HL-60 cells were cultured in the Eagle's Minimum Essential Medium $\left(10 \%\right.$ fetal calf serum) suspension at $37^{\circ} \mathrm{C}$ in $5.0 \% \mathrm{CO}_{2}$. The test material was dissolved in dimethyl sulfoxide (DMSO) to give a concentration of $10 \mathrm{mM}$, and the solution was diluted with the Essential Medium to give the concentrations of 200, 20 and $2.0 \mu \mathrm{mol}$, respectively. Each solution was combined with each cell suspension $(1 \times$ $10^{5}$ cells $/ \mathrm{ml}$ ) in the medium, respectively. After incubation at $37^{\circ} \mathrm{C}$ for 72 hours in $5.0 \% \mathrm{CO}_{2}$, the grown cells were labeled with $5.0 \mathrm{mg} / \mathrm{ml}$ MTT in phosphate buffer saline (PBS), and then absorbance of formazan dissolved by $20 \%$ sodium dodecyl sulfate (SDS) in $0.1 \mathrm{~N} \mathrm{HCl}$ was measured at $540 \mathrm{~nm}$ using microplate reader (Model 450, BIO-RAD). Each absorbance value was expressed as a percentage, relative to the control cell suspension which was prepared without the test substance by the same procedure as described above. All assays were performed three times, semilogarithmic plots were constructed from the averaged data, and the effective dose of the substance required to inhibit cell growth by $50 \%\left(\mathrm{IC}_{50}\right)$ was determined.

Acknowledgments We thank Dr. T. Yamori (Screening Committee of Anticancer Drugs supported by Grant-in-Aid for Scientific Research on Priority Area "Cancer" from the Ministry of Education, Culture, Sports, Science and Technology, Japan) for the measurement of the cytotoxic assay using a panel of 39 human cell lines. We are grateful to Ms M. Fujitake and Dr. K. Minoura of this university for the MS and NMR measurements, respectively. This Study was supported by a Grant-in-Aid for High Technology Research from the Ministry of Education, Culture, Sports, Science and Technology, Japan.

\section{References}

1. Iwamoto C, Yamada $\mathrm{T}$, Ito $\mathrm{Y}$, Minoura $\mathrm{K}$, Numata A. Cytotoxic cytochalasans from a Penicillium species separated from a marine alga. Tetrahedron 57: 2997-3004 (2001)

2. Yamada T, Iritani M, Doi M, Minoura K, Ito T, Numata A.
Absolute stereostructures of cell adhesion inhibitors, macrosphelides $\mathrm{C}, \mathrm{E} \sim \mathrm{G}$ and I, produced by a Periconia species separated from an Aplysia sea hare. J Chem Soc Perkin Trans 1: 3046-3053 (2001)

3. Yamada T, Iritani M, Minoura K, Numata A. Absolute stereostructures of cell adhesion inhibitors, macrosphelide $\mathrm{H}$ and L, from Periconia byssoides OUPS-N133. J Antibiot 55: 147-154 (2002)

4. Yamada $T$, Iritani M, Minoura K, Kawai K, Numata A. Peribysin $A \sim D$, potent cell-adhesion inhibitors from a sea hare-derived culture of Periconia species. J Org Biomol Chem 2131-2135 (2004)

5. Quang DN, Stadler M, Fournier J, Tomita A, Hashimoto T. Cohaerins $\mathrm{C} \sim \mathrm{F}$, four azaphilones from the xylariaceous fungus Annulohypoxylon cohaerens. Tetrahedron 62: 6349-6354 (2006)

6. Matsuzaki K, Tahara H, Inokoshi J, Tanaka H. New brominated and halogen-less derivatives and structureactivity relationship of azaphilaones inhibiting gp120-CD4 binding. J Antibiot 51: 1004-1011 (1998)

7. Yoshida E, Fujimoto H, Yamazaki M. Isolation of three new azaphilones, luteusins C, D, and E, from an Ascomycete, Talaromyces luteus. Chem Pharm Bull 44: 284-287 (1996)

8. Takahashi M, Koyama K, Natori S. Four new azphilones from Chaetomium globosum var. flavorviridae. Chem Pharm Bull 38: 625-628 (1990)

9. Toki S, Tanaka T, Uosaki Y, Yoshida M, Suzuki Y, Kita K, Mihara A, Ando K, Lokker NA, Giese NA, Matsuda Y. RP1551s, a family of azaphilones produced by Penicillium sp., inhibit the binding of PDGF to the extracellular domain of its receptor. J Antibiot 52: 235-244 (1999)

10. Yamada T, Doi M, Shigeta H, Muroga Y, Hosoe S, Numata A, Tanaka R. Absolute stereostructures of cytotoxic metabolites, chaetomugilins $\mathrm{A} \sim \mathrm{C}$, produced by $\mathrm{a}$ Chaetomium species separated from a marine fish. Tetrahedron Lett 49: 4192-4195 (2008)

11. Yamori T, Matsunaga A, Sato S, Yamazaki K, Komi A, Ishizu K, Mita I, Edatsugi H, Matsuba Y, Takezawa, K, Nakanishi O, Kohno H, Nakajima Y, Komatsu H, Andoh T, Tsuruo T. Potent antitumor activity of MS-247, a novel DNA minor groove binder, evaluated by an in vitro and in vivo human cancer cell line panel. Cancer Res 59: 4042-4049 (1999)

12. Yamori T. Panel of human cancer cell lines provides valuable database for drug discovery and bioinformatics. Cancer Chemother Pharmacol 52 (Suppl 1): S74-S79 (2003) 